\title{
EXISTENCE AND ASYMPTOTIC BEHAVIOR OF STANDING WAVES OF THE NONLINEAR HELMHOLTZ EQUATION IN THE PLANE
}

\author{
GILLES EVÉQUOZ
}

ABStRaCt. In this paper we study the semilinear elliptic problem

$$
-\Delta u-k^{2} u=Q|u|^{p-2} u \text { in } \mathbb{R}^{2},
$$

where $k>0, p \geq 6$ and $Q$ is a bounded function. We prove the existence of real-valued $W^{2, p}$-solutions, both for decaying and for periodic coefficient $Q$. In addition, a nonlinear far-field relation is derived for these solutions.

\section{INTRODUCTION AND MAIN RESULTS}

The purpose of this article is to study the existence and the properties of real-valued solutions of the semilinear problem

$$
-\Delta u+\lambda u=Q|u|^{p-2} u \quad \text { in } \mathbb{R}^{2}
$$

that vanish at infinity, in the case where $p>2$ and $Q: \mathbb{R}^{2} \rightarrow \mathbb{R}$ is a bounded function. For $\lambda \geq 0$, the problem (1) in $\mathbb{R}^{N}$ with such superlinear nonlinearities has received a great deal of attention, starting with the celebrated papers by Berestycki and Lions [6, 7. on the case $N \geq 3$ and by Berestycki, Gallouët and Kavian [5] for the case $N=2$. We refer the reader to the monographs [3, 19, 22, 24,26] and the references therein for a detailed account on the study of such equations. In contrast, much less is known about the case $\lambda<0$, due in particular to the fact that the usual variational method in $H^{1}\left(\mathbb{R}^{N}\right)$ breaks down, since the solutions of (11), if any, will not decay faster than $O\left(|x|^{\frac{1-N}{2}}\right.$ ) as $|x| \rightarrow \infty$ (see [17]). Recent results obtained by $\mathrm{T}$. Weth and the author [11] confirmed nevertheless the existence for $\lambda<0$ of nontrivial $W^{2, p}\left(\mathbb{R}^{N}\right)$-solutions for the problem (1) in $\mathbb{R}^{N}$ with $N \geq 3$. In a previous paper [10, existence results for (11) in all dimensions $N \geq 2$ and for more general nonlinearities were obtained by studying a Dirichlet-toNeumann boundary-value problem, but only nonlinearities having compact support were considered. Let us also mention results concerning complex-valued solutions of (11) with prescribed asymptotic behavior, obtained using contraction mapping arguments, by Gutiérrez [15] in dimension $N=3,4$ and with $p=4$, and by Jalade [16] in dimension $N=3$ for more general, compactly supported nonlinearities.

Our present goal is to extend the results of [1] to the two-dimensional case and, at the same time, to provide a basis for further study of the planar nonlinear Helmholtz equation. Without loss of generality, we shall focus on the case $\lambda=-1$ and therefore deal with the problem

$$
-\Delta u-u=Q|u|^{p-2} u \quad \text { in } \mathbb{R}^{2} .
$$

As in [11, we shall reformulate (2) as an integral equation, involving the resolvent operator $\mathscr{R}$ associated to the inhomogeneous Helmholtz equation

$$
-\Delta u-u=f \quad \text { in } \mathbb{R}^{2}
$$

and the outgoing radiation condition, which in two dimensions reads as:

$$
\nabla u(x) \cdot \frac{x}{|x|}-i u(x)=o\left(|x|^{-\frac{1}{2}}\right), \quad \text { as }|x| \rightarrow \infty
$$

(see [8, Chap. 3.4]). More precisely, we shall look for solutions of (2) that satisfy the fixed-point equation

$$
u=\mathbf{R}\left(Q|u|^{p-2} u\right), \quad u \in L^{p}\left(\mathbb{R}^{2}\right) .
$$

Here, $\mathbf{R}$ denotes the real part of the operator $\mathscr{R}$. For more details concerning the link between (4) and (2) we refer the reader to the introduction of [11.

2010 Mathematics Subject Classification. 35J20 (primary) 35J05 (secondary).

Key words and phrases. Nonlinear Helmholtz equation, standing waves, variational method, resolvent estimates, far-field expansion. 
Our first main result deals with the regularity and the asymptotic behavior of solutions of the nonlinear problem (4). There, and in the sequel, $\mathscr{F}$ denotes the Fourier transform on the space of tempered distributions.

Theorem 1.1. Let $6 \leq p<\infty, Q \in L^{\infty}\left(\mathbb{R}^{2}\right)$ and consider a solution $u$ of (4). Then, $u \in W^{2, q}\left(\mathbb{R}^{2}\right)$ for all $6 \leq q<\infty$ and it is a strong solution of (2).

Moreover, if $p>6$, we have $u \in W^{2, q}\left(\mathbb{R}^{2}\right)$ for all $4<q<\infty$ and

$$
u(x)=\sqrt{\frac{\pi}{2}}|x|^{-\frac{1}{2}} \operatorname{Re}\left[e^{i|x|+\frac{i \pi}{4}} \mathfrak{f}_{u}\left(\frac{x}{|x|}\right)\right]+o\left(|x|^{-\frac{1}{2}}\right), \text { as }|x| \rightarrow \infty,
$$

where $\mathfrak{f}_{u}(\xi)=\mathscr{F}\left(Q|u|^{p-2} u\right)(\xi)$ for $\xi \in \mathbb{R}^{2}$ with $|\xi|=1$.

Remark 1.2. As for the Helmholtz equation in dimension 3 (see [11, p. 694]), the pointwise expansion (5) is satisfied for all noncritical exponents $p \in(6, \infty)$. In the case $p=6$, it holds for radial solutions, under additional assumptions on the function $Q$. Indeed, assuming $Q$ to be $C^{1}$, radially symmetric and radially decreasing, we find by [10, Theorem 4], that every radial solution of (4) satisfies $|u(x)| \leq C|x|^{-\frac{1}{2}}$. From Proposition 2.2 below, we then obtain (5). In general, however, only the following weaker form of (5) holds (cf. [11, Lemma 4.3]):

$$
\left.\left.\lim _{R \rightarrow \infty} \frac{1}{R} \int_{B_{R}(0)}\left|u(x)-\sqrt{\frac{\pi}{2}}\right| x\right|^{-\frac{1}{2}} \operatorname{Re}\left[e^{i|x|+\frac{i \pi}{4}} \mathfrak{f}_{u}\left(\frac{x}{|x|}\right)\right]\right|^{2} d x=0 .
$$

Our second main result concerns the existence of solutions for (4), and hence for (2), under two different assumptions on the nonnegative function $Q$.

Theorem 1.3. For $6 \leq p<\infty$ and $Q \in L^{\infty}\left(\mathbb{R}^{2}\right) \backslash\{0\}, Q \geq 0$, the following holds.

(a) If $Q(x) \rightarrow 0$ as $|x| \rightarrow \infty$, the problem (4) admits a sequence of pairs of solutions $\pm u_{n} \in W^{2, q}\left(\mathbb{R}^{2}\right)$, for all $6 \leq q<\infty$ if $p=6$ and all $4<q<\infty$ if $p>6$, such that $\left\|u_{n}\right\|_{p} \rightarrow \infty$ as $n \rightarrow \infty$.

(b) If $Q$ is $\mathbb{Z}^{2}$-periodic and $p>6$, then (4) has a nontrivial solution pair $\pm u \in W^{2, q}\left(\mathbb{R}^{2}\right)$ for all $4<q<\infty$.

Remark 1.4. Theorem 1.1 and 1.3 can be extended to more general nonlinearities, like those studied in [9] for $N \geq 3$. Also, replacing the assumption $Q(x) \rightarrow 0$ as $|x| \rightarrow \infty$ by $Q \in L^{\frac{3}{2}}\left(\mathbb{R}^{2}\right) \cap L^{\infty}\left(\mathbb{R}^{2}\right)$ in Theorem 1.3 (a), one can prove that for every $p \in(2, \infty)$ the problem (4) has infinitely many pairs of solutions $\left\{ \pm u_{n}\right\}$ such that $Q^{\frac{1}{p}} u_{n} \in L^{p}\left(\mathbb{R}^{2}\right)$ for all $n$ and $\int_{\mathbb{R}^{2}} Q\left|u_{n}\right|^{p} d x \rightarrow \infty$ as $n \rightarrow \infty$.

The proof of the above results is based on the method developed in the recent paper [11, but we emphasize that these results do not follow from their higher-dimensional counterparts. Indeed, the presence of a logarithmic singularity at 0 in the kernel of the resolvent operator in $\mathbb{R}^{2}$ (cf. [8]) requires new estimates, different from those obtained in [11, and which we believe to be also of independent interest.

The paper is organized as follows. In the next section, we define the resolvent Helmholtz operator $\mathscr{R}$ and derive $L^{p}$-estimates similar to [15, 18. Next, the asymptotic expansion and the decay of solutions of linear equations are studied and the section concludes with the proof of Theorem 1.1. Section 3 is devoted to the existence proof for solutions of (4). It starts with the extension of the dual variational method of 11 to $\mathbb{R}^{2}$ and continues with the proof of Theorem 1.3(a), as an application of the symmetric Mountain Pass Theorem. There, an interaction estimate, more involved than in the case $N \geq 3$ is used to construct finite-dimensional subspaces of arbitrary dimension on which the quadratic part of the energy functional is positive. Next, the periodic case is studied and a nonvanishing property for the quadratic form associated to the resolvent $\mathscr{R}$ is derived. As in [11, it constitutes a key ingredient in the proof of the existence of solutions in the periodic case, by which the paper concludes.

\section{THE PLANAR RESOLVENT AND THE FAR-FIELD RELATION}

For a function $f$ in the Schwartz space $\mathscr{S}\left(\mathbb{R}^{2}\right)$, the unique solution of the Helmholtz equation $-\Delta u-u=$ $f$ in $\mathbb{R}^{2}$ which satisfies the radiation condition (3) is given by the convolution $u=\Phi * f$, where

$$
\Phi(x)=\frac{i}{4} H_{0}^{(1)}(|x|), \quad x \in \mathbb{R}^{2} .
$$


Here, $H_{0}^{(1)}$ denotes the Hankel function of the first kind of order 0 (see e.g., 8, Chap. 3.4]). In view of the asymptotic behavior of $\Phi$ given by

$$
\Phi(x)= \begin{cases}\frac{1}{2 \sqrt{2 \pi}}|x|^{-\frac{1}{2}} e^{i|x|+i \frac{\pi}{4}}\left[1+O\left(|x|^{-1}\right)\right] & \text { as }|x| \rightarrow \infty, \\ \frac{1}{2 \pi} \log \left(\frac{2}{|x|}\right)\left[1+O\left(\frac{1}{|\log | x||}\right)\right] & \text { as } x \rightarrow 0,\end{cases}
$$

(see, e.g., 20, Eq. (5.16.3)]), there is a constant $C_{0}>0$ such that

$$
|\Phi(x)| \leq C_{0} \min \left\{1+|\log | x||,|x|^{-\frac{1}{2}}\right\} \quad \text { for } x \in \mathbb{R}^{2} \backslash\{0\} .
$$

Following ideas of Kenig-Ruiz and Sogge [18, Theorem 2.3] and Gutiérrez [15, Theorem 6], we prove estimates which show that the resolvent Helmholtz operator $f \mapsto \Phi * f$, defined for $f \in \mathscr{S}\left(\mathbb{R}^{2}\right)$, has for certain $1 \leq t, q \leq \infty$ a continuous extension $\mathscr{R}: L^{t}\left(\mathbb{R}^{2}\right) \rightarrow L^{q}\left(\mathbb{R}^{2}\right)$. In the following, given $1 \leq p \leq \infty$, we let $p^{\prime}$ denote its conjugate exponent.

Theorem 2.1. Let $1 \leq t<\frac{4}{3}$ and $4<q \leq \infty$ satisfy $\frac{2}{3} \leq \frac{1}{t}-\frac{1}{q}<1$. There is a constant $C=C(t, q)>0$ such that

$$
\|\mathscr{R} f\|_{q} \leq C\|f\|_{t} \quad \text { for all } f \in \mathscr{S}\left(\mathbb{R}^{2}\right) .
$$

In particular, if $6 \leq q<\infty$ then (8) holds with $t=q^{\prime}$.

In order to prove the above estimates, we consider a decomposition of the fundamental solution $\Phi$ which we shall use also further below (see Theorem 3.1).

Fix $\psi \in \mathscr{S}\left(\mathbb{R}^{2}\right)$ such that the Fourier transform $\widehat{\psi} \in \mathscr{C}_{c}^{\infty}\left(\mathbb{R}^{2}\right)$ is radial, $0 \leq \widehat{\psi} \leq 1, \widehat{\psi}(\xi)=1$ for ||$\xi|-1| \leq \frac{1}{6}$ and $\widehat{\psi}(\xi)=0$ for ||$\xi|-1| \geq \frac{1}{4}$. Write $\Phi=\Phi_{1}+\Phi_{2}$, where

$$
\Phi_{1}:=2 \pi(\psi * \Phi) \text {, and } \Phi_{2}=\Phi-\Phi_{1} .
$$

Since $\psi$ is a Schwartz function, we obtain from (7), making $C_{0}$ larger if necessary,

$$
\left|\Phi_{1}(x)\right| \leq C_{0}(1+|x|)^{-\frac{1}{2}}, \quad x \in \mathbb{R}^{2} .
$$

On the other hand, since $\mathscr{F}(\Phi)(\xi)=\frac{1}{2 \pi}\left(|\xi|^{2}-1-i 0\right)^{-1}$ as a tempered distribution (see [12]) and since $\mathscr{F}\left(\Phi_{2}\right)=(1-\widehat{\psi}) \mathscr{F}(\Phi)$, it follows that $\mathscr{F}\left(\Phi_{2}\right) \in C^{\infty}\left(\mathbb{R}^{2}\right)$ and $\mathscr{F}\left(\Phi_{2}\right)(\xi)=\left(|\xi|^{2}-1\right)^{-1}$ for $|\xi| \geq \frac{5}{4}$. Consequently, $\partial^{\gamma} \mathscr{F}\left(\Phi_{2}\right) \in L^{1}\left(\mathbb{R}^{2}\right)$ for all $\gamma \in \mathbb{N}_{0}^{N}$ such that $|\gamma| \geq 1$ and this gives $\left|\Phi_{2}(x)\right| \leq \kappa_{s}|x|^{-s}$ for all $s>0$, with some constant $\kappa_{s}>0$. Using also (7) and (10), we obtain, making again $C_{0}$ larger,

$$
\left|\Phi_{2}(x)\right| \leq C_{0} \min \left\{1+|\log | x||,|x|^{-3}\right\}, \quad x \in \mathbb{R}^{2} \backslash\{0\} .
$$

Proof of Theorem [2.1. The proof is inspired by Theorem 6 in [15]. In the sequel, $C$ will denote a constant, whose value may change from line to line.

Using (11) we see that $\Phi_{2} \in L^{r}\left(\mathbb{R}^{2}\right)$ for all $1 \leq r<\infty$, and therefore Young's inequality gives for $1 \leq t, q \leq \infty$ such that $0 \leq \frac{1}{t}-\frac{1}{q}<1$,

$$
\left\|\Phi_{2} * f\right\|_{q} \leq\left\|\Phi_{2}\right\|_{r}\|f\|_{t} \leq C\|f\|_{t} \quad \text { for all } f \in \mathscr{S}\left(\mathbb{R}^{2}\right) .
$$

To estimate the convolution with $\Phi_{1}$, let us fix a radial, nonnegative function $\eta \in C_{c}^{\infty}\left(\mathbb{R}^{2}\right)$ such that $\eta(x)=1$ if $0 \leq|x| \leq 1, \eta(x)=0$ if $|x| \geq 2$. For $j \in \mathbb{N}$, define $\varphi_{j} \in C_{c}^{\infty}\left(\mathbb{R}^{2}\right)$ by $\varphi_{j}(x)=$ $\eta\left(x / 2^{j}\right)-\eta\left(x / 2^{j-1}\right)$. Let also $\varphi_{0}=\eta$. We then have the dyadic decomposition

$$
\Phi_{1}=\sum_{j=0}^{\infty} \Phi_{1}^{j} \quad \text { with } \Phi_{1}^{j}:=\Phi_{1} \varphi_{j} \text { for } j \in \mathbb{N} \cup\{0\} .
$$

Choosing also $\varphi \in \mathscr{S}\left(\mathbb{R}^{2}\right)$ such that its Fourier transform $\widehat{\varphi} \in C_{c}^{\infty}\left(\mathbb{R}^{2}\right)$ is radial, nonnegative and satisfies $\widehat{\varphi}(\xi)=1$ on $\left\{\xi:|| \xi|-1| \leq \frac{1}{2}\right\}$ and $\widehat{\varphi}(\xi)=0$ on $\left\{\xi:|| \xi|-1| \geq \frac{3}{4}\right\}$, we see that $\left(\Phi_{1} * \varphi\right) * f=2 \pi \Phi_{1} * f$ for all $f \in \mathscr{S}\left(\mathbb{R}^{2}\right)$, since supp $\mathscr{F}\left(\Phi_{1}\right) \subset\left\{\xi:|| \xi|-1| \leq \frac{1}{4}\right\}$. Hence, we look at the decomposition

$$
\Phi_{1} * \varphi=\sum_{j=0}^{\infty} Q^{j} \quad \text { with } Q^{j}:=\Phi_{1}^{j} * \varphi \text { for } j \in \mathbb{N} \cup\{0\} .
$$


From the decay properties of $\Phi_{1}$, we see that

$$
\left\|Q^{j}\right\|_{\infty} \leq\|\varphi\|_{1}\left\|\Phi_{1}^{j}\right\|_{\infty} \leq C 2^{-\frac{j}{2}} \quad \text { for all } j \geq 1,
$$

where $C$ is independent of $j$. On the other hand, Plancherel's identity and the Stein-Tomas Theorem [25] imply for $1 \leq t \leq \frac{6}{5}$ and $f \in \mathscr{S}\left(\mathbb{R}^{2}\right)$,

$$
\begin{aligned}
\left\|Q^{j} * f\right\|_{2}^{2} & =(2 \pi)^{2} \int_{\| \xi|-1| \leq \frac{3}{4}}\left|\widehat{\Phi}_{1}^{j}(\xi) \widehat{g}(\xi)\right|^{2} d \xi \leq C \int_{\frac{1}{4}}^{\frac{7}{4}} r^{\frac{4}{t}-3}\left|\widehat{\Phi}_{1}^{j}(r)\right|^{2}\|g\|_{t}^{2} d r \\
& \leq C\|\varphi\|_{1}^{2}\|f\|_{t}^{2} \int_{\mathbb{R}^{2}}\left|\Phi_{1}^{j}(x)\right|^{2} d x \leq C 2^{j}\|f\|_{t}^{2},
\end{aligned}
$$

where we have set $g=\varphi * f$, and $C$ does not depend on $j$. From these two estimates and the Riesz-Thorin theorem [23, Theorem V.1.3], it follows that

$$
\left\|Q^{j} * f\right\|_{q} \leq C 2^{j\left(\frac{2}{q}-\frac{1}{2}\right)}\|f\|_{t} \quad \text { for all } f \in \mathscr{S}\left(\mathbb{R}^{2}\right),
$$

$1 \leq t \leq \frac{6}{5}$ and $2 \leq q \leq \frac{t^{\prime}}{3}$. Observe that the exponent is negative if $q>4$. Since $\Phi_{0}^{j} \in L^{1}\left(\mathbb{R}^{2}\right) \cap L^{\infty}\left(\mathbb{R}^{2}\right)$, we conclude that for $1 \leq t<\frac{12}{11}$ and $4<q \leq \frac{t^{\prime}}{3}$,

$$
\left\|\Phi_{1} * f\right\|_{q}=\frac{1}{2 \pi}\left\|\left(\Phi_{1} * \varphi\right) * f\right\|_{q} \leq \sum_{j=0}^{\infty}\left\|Q^{j} * f\right\|_{q} \leq C\|f\|_{t} \sum_{j=0}^{\infty} 2^{j\left(\frac{2}{q}-\frac{1}{2}\right)},
$$

and therefore

$$
\left\|\Phi_{1} * f\right\|_{q} \leq C\|f\|_{t} .
$$

By duality and convexity, this estimate holds for all $1 \leq t<\frac{4}{3}$ and $4<q \leq \infty$ such that $\frac{1}{t}-\frac{1}{q}>\frac{2}{3}$. Taking into account the estimate (12) for $\Phi_{2}$, we obtain (8) for all $1 \leq t<\frac{4}{3}, 4<q \leq \infty$ such that $\frac{2}{3}<\frac{1}{t}-\frac{1}{q}<1$.

To conclude the proof, it remains to show that (16) also holds when $\frac{1}{t}-\frac{1}{q}=\frac{2}{3}$. We proceed similarly to [15. Theorem 6]. Using real interpolation (see [23, Section V.3]), it is enough to prove the restricted weak-type estimates

$$
\left\|\Phi_{1} * f\right\|_{q, \infty} \leq C\|f\|_{t, 1} \quad \text { for all } f \in \mathscr{S}\left(\mathbb{R}^{2}\right)
$$

for the endpoints $(t, q)=\left(\frac{12}{11}, 4\right)$ and $\left(\frac{4}{3}, 12\right)$, where $\|\cdot\|_{r, s}$ denotes the norm on the Lorentz space $L^{r, s}\left(\mathbb{R}^{2}\right)$. Moreover, by [23, Theorem V.3.13 and Theorem V.3.21], it suffices to prove (17) for characteristic functions of measurable sets:

$$
\lambda\left|\left\{x \in \mathbb{R}^{2}:\left|\left(\Phi_{1} * 1_{E}\right)(x)\right|>\lambda\right\}\right|^{\frac{1}{q}} \leq C|E|^{\frac{1}{t}} \quad \text { for all } \lambda>0,|E|<\infty .
$$

Here, $|E|$ denotes the measure of $E \subset \mathbb{R}^{2}$ and $1_{E}$ its characteristic function. Setting $A:=\left\{x \in \mathbb{R}^{2}\right.$ : $\left.\left|\left(\Phi_{1} * 1_{E}\right)(x)\right|>\lambda\right\}$, choosing $\varphi \in \mathscr{S}\left(\mathbb{R}^{2}\right)$ as above and recalling the dyadic decomposition (13), we can write

$$
|A|=\int_{A} d x \leq \frac{1}{\lambda} \int_{\mathbb{R}^{2}}\left|\left(\Phi_{1} * 1_{E}\right)(x)\right| 1_{A}(x) d x \leq \frac{1}{\lambda} \sum_{j=0}^{\infty} \int_{\mathbb{R}^{2}}\left|\left(Q^{j} * 1_{E}\right)(x)\right| 1_{A}(x) d x .
$$

From the estimate (15) with $t=\frac{6}{5}$ and by duality, we obtain

$$
\left\|Q^{j} * f\right\|_{2} \leq C 2^{\frac{j}{2}}\|f\|_{\frac{6}{5}} \quad \text { and } \quad\left\|Q^{j} * f\right\|_{6} \leq C 2^{\frac{j}{2}}\|f\|_{2}
$$

for all $f \in \mathscr{S}\left(\mathbb{R}^{2}\right)$, the constant $C$ being independent of $j$. Moreover, (14) gives

$$
\left\|Q^{j} * f\right\|_{\infty} \leq C 2^{-\frac{j}{2}}\|f\|_{1}, \quad j \geq 1 .
$$

For $M \in \mathbb{N}_{0}$, we therefore obtain, using Hölder's inequalityand approximating $1_{E}$ by Schwartz functions,

$$
\begin{aligned}
\sum_{j=0}^{\infty} \int_{\mathbb{R}^{2}}\left|\left(Q^{j} * 1_{E}\right)(x)\right| 1_{A}(x) d x & \leq \sum_{j=0}^{M}\left\|Q^{j} * 1_{E}\right\|_{2}|A|^{\frac{1}{2}}+\sum_{j=M+1}^{\infty}\left\|Q^{j} * 1_{E}\right\|_{\infty}|A| \\
& \leq C\left\{2^{\frac{M}{2}}|E|^{\frac{5}{6}}|A|^{\frac{1}{2}}+2^{-\frac{M+1}{2}}|E \| A|\right\} .
\end{aligned}
$$


Choosing $M \in \mathbb{N}_{0}$ with $2^{\frac{M}{2}} \leq|E|^{\frac{1}{12}}|A|^{\frac{1}{4}} \leq 2^{\frac{M+1}{2}}$, the preceding estimates give

$$
\lambda|A| \leq \sum_{j=0}^{\infty} \int_{\mathbb{R}^{2}}\left|\left(Q^{j} * 1_{E}\right)(x)\right| 1_{A}(x) d x \leq C|E|^{\frac{11}{12}}|A|^{\frac{3}{4}}
$$

which yields $\lambda|A|^{\frac{1}{4}} \leq C|E|^{\frac{11}{12}}$ and shows (18) for the exponents $t=\frac{12}{11}, q=4$.

Similarly, Hölder's inequality and the estimates (19), (20) give

$$
\begin{aligned}
\sum_{j=0}^{\infty} \int_{\mathbb{R}^{2}}\left|\left(Q^{j} * 1_{E}\right)(x)\right| 1_{A}(x) d x & \leq \sum_{j=0}^{M}\left\|Q^{j} * 1_{E}\right\|_{6}|A|^{\frac{5}{6}}+\sum_{j=M+1}^{\infty}\left\|Q^{j} * 1_{E}\right\|_{\infty}|A| \\
& \leq C\left\{2^{\frac{M}{2}}|E|^{\frac{1}{2}}|A|^{\frac{5}{6}}+2^{-\frac{M+1}{2}}|E \| A|\right\}
\end{aligned}
$$

Choosing this time $M \in \mathbb{N}_{0}$ such that $2^{\frac{M}{2}} \leq|E|^{\frac{1}{4}}|A|^{\frac{1}{12}} \leq 2^{\frac{M+1}{2}}$, we find that $\lambda|A|^{\frac{1}{12}} \leq C|E|^{\frac{3}{4}}$, proving (18) for the exponents $p=\frac{4}{3}, q=12$. The proof is complete.

We note that the conclusion of Theorem 2.1 is false for $(t, q)=(1, \infty)$. Indeed, if $f \in C_{c}^{\infty}\left(\mathbb{R}^{2}\right) \backslash\{0\}$ is nonnegative and $f(x)=0$ for $|x| \geq 1$, consider the sequence $\left(f_{k}\right)_{k}$, where $f_{k}(x)=k^{2} f(k x), x \in \mathbb{R}^{2}$. Then, $\left\|f_{k}\right\|_{1}=\|f\|_{1}$ for all $k$, but for every $x \neq 0$, we find

$$
\left(\Phi * f_{k}\right)(x)=\int_{B_{1}(0)} \Phi\left(x-k^{-1} y\right) f(y) d y \longrightarrow \Phi(x)\|f\|_{1}, \quad \text { as } k \rightarrow \infty
$$

by the Dominated Convergence Theorem. Hence, $\left\|\Phi * f_{k}\right\|_{\infty} \rightarrow \infty$ as $k \rightarrow \infty$.

We now turn to the pointwise asymptotic expansion of solutions of the Helmholtz equation and first look at the the linear problem $u=\mathscr{R} f$.

Proposition 2.2. Let $f \in L^{1}\left(\mathbb{R}^{2}\right)$ satisfy $|f(x)| \leq \kappa|x|^{-2-\varepsilon}$ for some $\kappa, \varepsilon>0$. Then

$$
\mathscr{R} f(x)=\sqrt{\frac{\pi}{2}} \frac{e^{i|x|+\frac{i \pi}{4}}}{|x|^{\frac{1}{2}}} \widehat{f}\left(\frac{x}{|x|}\right)+o\left(|x|^{-\frac{1}{2}}\right), \quad \text { as }|x| \rightarrow \infty .
$$

Proof. Consider first for $x \in \mathbb{R}^{2}$ with $|x| \geq 2$,

$$
I_{1}(x)=\int_{B_{1}(x)} \Phi(x-y) f(y) d y .
$$

From (7), we see that $|\Phi(z)| \leq C_{0}(1+|\log | z||)$ for all $|z| \leq 1$, and we can write

$$
\begin{aligned}
\left|I_{1}(x)\right| & \leq C_{0} \kappa \int_{B_{1}(x)}(1+|\log | x-y||)|y|^{-2-\varepsilon} d y \\
& \leq C_{0} \kappa\left(\frac{|x|}{2}\right)^{-2-\varepsilon} \int_{B_{1}(0)}(1+|\log | y||) d y
\end{aligned}
$$

where the last integral is finite. In particular, $I_{1}(x)=o\left(|x|^{-\frac{1}{2}}\right)$ as $|x| \rightarrow \infty$. Next, let $A(x)=\left\{y \in \mathbb{R}^{2}\right.$ : $|x-y|>1$ and $|y| \geq \sqrt{|x|}\}$ and consider

$$
I_{2}(x)=\int_{A(x)} \Phi(x-y) f(y) d y
$$


The estimate (7) implies $|\Phi(z)| \leq C_{0}|z|^{-\frac{1}{2}}$ for all $|z|>1$, and therefore

$$
\begin{aligned}
\left|I_{2}(x)\right| & \leq C_{0} \int_{A(x)}|x-y|^{-\frac{1}{2}}|f(y)| d y \\
& \leq C_{0}|x|^{-\frac{1}{2}} \int_{A(x)}\left(1+|x-y|^{-\frac{1}{2}}|y|^{\frac{1}{2}}\right)|f(y)| d y \\
& \leq C_{0}|x|^{-\frac{1}{2}}\left(\int_{A(x)}|f(y)| d y+\kappa \int_{A(x)}|x-y|^{-\frac{1}{2}}|y|^{-\frac{3}{2}-\varepsilon} d y\right) .
\end{aligned}
$$

Since $f \in L^{1}\left(\mathbb{R}^{2}\right)$, the first integral on the last line goes to zero uniformly as $|x| \rightarrow \infty$. The same is true for the second integral, since $A(x) \subset \mathbb{R}^{2} \backslash B_{1}(0)$ and since $-\frac{1}{2}+\left(-\frac{3}{2}-\varepsilon\right)<-2$ (see, e.g., 2, Appendix 2, Lemma 1]). Hence, $I_{2}(x)=o\left(|x|^{-\frac{1}{2}}\right)$ as $|x| \rightarrow \infty$. Concerning the remaining integral

$$
I_{3}(x)=\int_{D(x)} \Phi(x-y) f(y) d y,
$$

where $D(x)=\left\{y \in \mathbb{R}^{2}:|x-y|>1\right.$ and $\left.|y| \leq \sqrt{|x|}\right\}$, we can write using (6),

$$
I_{3}(x)=\frac{e^{\frac{i \pi}{4}}}{2 \sqrt{2 \pi}} \int_{D(x)} \frac{e^{i|x-y|}}{|x-y|^{\frac{1}{2}}}(1+\delta(|x-y|)) f(y) d y,
$$

where $\sup _{r \geq 1} r|\delta(r)|<\infty$. Furthermore, setting $\widehat{x}:=\frac{x}{|x|}$ for $x \neq 0$, one finds

$$
|| x-y|-| x|+\widehat{x} \cdot y| \leq|x|^{-1}|y|^{2} \quad \text { for all } x, y \in \mathbb{R}^{2} \text { with } x \neq 0 \text { and }|y| \leq \frac{|x|}{2} .
$$

Arguing as in [11, Proposition 2.8] and using the estimate $|f(y)| \leq \kappa|y|^{-2-\varepsilon}$, we obtain

$$
\left|I_{3}(x)-\frac{1}{2 \sqrt{2 \pi}} \frac{e^{i|x|+i \frac{\pi}{4}}}{|x|^{\frac{1}{2}}} \int_{D(x)} e^{-i \widehat{x} \cdot y} f(y) d y\right| \leq \tilde{\kappa}|x|^{-\frac{1}{2}-\frac{\varepsilon}{4}},
$$

for some constant $\tilde{\kappa}>0$. Putting together the estimates for $I_{1}, I_{2}$ and $I_{3}$ and using the integrability of $f$, we deduce that

$$
\mathscr{R} f(x)=I_{1}(x)+I_{2}(x)+I_{3}(x)=\sqrt{\frac{\pi}{2}} \frac{e^{i|x|+i \frac{\pi}{4}}}{|x|^{\frac{1}{2}}} \widehat{f}(\widehat{x})+o\left(|x|^{-\frac{1}{2}}\right), \quad \text { as }|x| \rightarrow \infty,
$$

and this concludes the proof.

The next result gives an upper bound for the decay of solutions of convolution equations involving a kernel with the asymptotic properties of $\Phi$. Combined with the regularity result below, it will provide a decay bound for solutions of the nonlinear problem (4).

Lemma 2.3. Let $u, V: \mathbb{R}^{2} \rightarrow \mathbb{R}$ be measurable functions satisfying $V \in L^{q}\left(\mathbb{R}^{2}\right), V u \in L^{\tilde{q}}\left(\mathbb{R}^{2}\right)$, where $1<q, \tilde{q}<\frac{4}{3}$. If $u=K *(V u)$ and

$$
|K(x)| \leq C_{0} \min \left\{1+|\log | x||,|x|^{-\frac{1}{2}}\right\} \quad \text { for } x \neq 0,
$$

then there exists a constant $C>0$ such that $|u(x)| \leq C|x|^{-\frac{1}{2}}$ for all $x \neq 0$.

Proof. Let $B_{R}:=B_{R}(0)$ and $M_{R}:=\mathbb{R}^{2} \backslash B_{R}$ for $R>0$, and define $\widetilde{K}(x)=C_{0} \min \left\{1+|\log | x||,|x|^{-\frac{1}{2}}\right\}$ for $x \neq 0$. Hölder's inequality, then gives

$$
\begin{aligned}
& \int_{M_{R}} \widetilde{K}(x-y)|V(y)| d y \leq C_{0} \int_{M_{R}}|V(y)| \min \left\{1+|\log | x-y||,|x-y|^{-\frac{1}{2}}\right\} d y \\
& \leq C_{0}\left(\int_{M_{R}}|V(y)|^{q}\right)^{\frac{1}{q}}\left(\int_{B_{1}(0)}(1+|\log | y||)^{q^{\prime}} d y+\int_{\mathbb{R}^{2} \backslash B_{1}(0)}|y|^{\frac{q^{\prime}}{2}} d y\right)^{\frac{1}{q^{\prime}}}
\end{aligned}
$$


which, as $R \rightarrow \infty$, tends to 0 uniformly in $x$, since $4<q^{\prime}<\infty$. Hence, we may fix $R>1$ such that

$$
\sup _{x \in \mathbb{R}^{2}} \int_{M_{R}} \tilde{K}(x-y)|V(y)| d y<\frac{1}{4} .
$$

The decay estimate on $u$ will follow with the help of an iteration procedure similar to the one of Zemach and Odeh [27]. For $|x| \geq R$ we set

$$
u_{0}(x)=\int_{B_{R}} K(x-y) V(y) u(y) d y, \quad B_{0}(x)=\int_{M_{R}} K(x-y) V(y) u(y) d y,
$$

and define inductively for $k \geq 1$,

$$
u_{k}(x)=\int_{M_{R}} K(x-y) V(y) u_{k-1}(y) d x, B_{k}(x)=\int_{M_{R}} K(x-y) V(y) B_{k-1}(y) d x .
$$

Thus, for each $m \in \mathbb{N}$,

$$
u=\sum_{k=0}^{m} u_{k}+B_{m}
$$

Since $V u \in L^{\tilde{q}}\left(\mathbb{R}^{2}\right)$ with $1<\tilde{q}<\frac{4}{3}$, and $|K(x)| \leq \widetilde{K}(x)$ for all $x$, we find that $\beta_{0}:=\sup _{|x|>R}\left|B_{k}(x)\right|<\infty$. Moreover, setting $\beta_{k}:=\sup _{|x|>R}\left|B_{k}(x)\right|$, (22) yields $\beta_{k} \leq \frac{1}{4} \beta_{k-1}$, for all $k \geq 1$, and therefore $\beta_{k} \rightarrow 0$, as $k \rightarrow \infty$. This gives

$$
u=\sum_{k=0}^{\infty} u_{k}, \quad \text { uniformly in } M_{R} .
$$

Moreover, since $u_{0} \in L^{\infty}\left(\mathbb{R}^{2}\right)$ and $V u \in L^{1}\left(B_{R}\right)$, we have $\mu_{0}:=\sup _{|x| \geq R}|x|^{\frac{1}{2}}\left|u_{0}(x)\right|<\infty$. Setting, for $k \geq 1$, $\mu_{k}:=\sup _{|x| \geq R}|x|^{\frac{1}{2}}\left|u_{k}(x)\right|$, and noticing that $\widetilde{K}(z)=C_{0}|z|^{-\frac{1}{2}}$ for all $|z| \geq 1$, we obtain

$$
\begin{aligned}
|x|^{\frac{1}{2}}\left|u_{k}(x)\right| & \leq \mu_{k-1}|x|^{\frac{1}{2}} \int_{M_{R}} \widetilde{K}(x-y)|V(y)||y|^{-\frac{1}{2}} d y \\
& \leq \mu_{k-1}|x|^{\frac{1}{2}}\left[C_{0}\left(\frac{|x|}{2}\right)^{-\frac{1}{2}} \int_{R \leq|y| \leq \frac{|x|}{2}}|V(y)||y|^{-\frac{1}{2}} d y\right. \\
& \left.+\left(\frac{|x|}{2}\right)^{-\frac{1}{2}} \int_{|y| \geq \max \left\{R, \frac{|x|}{2}\right\}} \widetilde{K}(x-y)|V(y)| d y\right] \\
& \leq 2 \sqrt{2} \mu_{k-1} \sup _{z \in \mathbb{R}^{2}} \int_{M_{R}} \widetilde{K}(z-y)|V(y)| d y,
\end{aligned}
$$

for all $|x| \geq R$, and from (22), we deduce that $\mu_{k} \leq \frac{\mu_{k-1}}{\sqrt{2}}$. As a consequence,

$$
\sup _{|x| \geq R}|x|^{\frac{1}{2}}|u(x)| \leq \sum_{k=0}^{\infty} \mu_{k} \leq \mu_{0} \sum_{k=0}^{\infty} 2^{-\frac{k}{2}}<\infty,
$$

and this concludes the proof.

The last preliminary step towards the proof of Theorem 1.1 consists in establishing regularity properties for the solutions of (4). This will also prove the first part of Theorem 1.1.

Lemma 2.4. Let $6 \leq p<\infty, Q \in L^{\infty}\left(\mathbb{R}^{2}\right)$ and consider a solution $u \in L^{p}\left(\mathbb{R}^{2}\right)$ of (4). Then $u$ is a strong solution of (2) and it belongs to $W^{2, q}\left(\mathbb{R}^{2}\right)$ for all $6 \leq q<\infty$, if $p=6$, and all $4<q<\infty$, if $p>6$.

Proof. Since $Q \in L^{\infty}\left(\mathbb{R}^{2}\right)$ and $6 \leq p<\infty$, we find that $f:=Q|u|^{p-2} u$ belongs to $L^{p^{\prime}}\left(\mathbb{R}^{2}\right)$ and that $1<p^{\prime} \leq \frac{6}{5}$. Hence, Theorem 2.1 gives $u \in L^{6}\left(\mathbb{R}^{2}\right) \cap L^{\infty}\left(\mathbb{R}^{2}\right)$, and since $p-1 \geq 5$, this means that $f$ belongs to $L^{\frac{6}{5}}\left(\mathbb{R}^{2}\right) \cap L^{\infty}\left(\mathbb{R}^{2}\right)$. Thus, we obtain from elliptic estimates (see [14, Chapter 9]) that $u \in W^{2, \sigma}\left(\mathbb{R}^{2}\right)$ for all $6 \leq \sigma<\infty$. This proves the lemma in the case $p=6$.

Assuming next $p>6$, we claim that $u \in L^{q}\left(\mathbb{R}^{2}\right)$ for all $4<q \leq \infty$. Supposing for the moment that it is true, we find $f=Q|u|^{p-2} u \in L^{t}\left(\mathbb{R}^{2}\right)$ for all $\frac{4}{p-1}<t \leq \infty$, where $p-1>1$. Hence, we get from elliptic estimates that $u \in W^{2, q}\left(\mathbb{R}^{2}\right)$ for all $4<q<\infty$ and the lemma is proved. 
We now prove the claim. As a consequence of the first step, we find that $f \in L^{p^{\prime}}\left(\mathbb{R}^{2}\right) \cap L^{\infty}\left(\mathbb{R}^{2}\right)$. Applying Theorem 2.1 with $t=p^{\prime}<\frac{6}{5}$, and using the fact that $u \in L^{\infty}\left(\mathbb{R}^{2}\right)$, we obtain that $u \in L^{q}\left(\mathbb{R}^{2}\right)$ for all $4<q \leq \infty$ such that $\frac{1}{q} \leq \frac{1}{p^{\prime}}-\frac{2}{3}$. If $\frac{1}{p^{\prime}}-\frac{2}{3} \geq \frac{1}{4}$, the claim follows. Otherwise, we iterate the argument and find that $f \in L^{t}\left(\mathbb{R}^{2}\right)$ for all $1 \leq t \leq \infty$ such that $\frac{1}{t} \leq(p-1)\left(\frac{1}{p^{\prime}}-\frac{2}{3}\right)$. Let $\frac{1}{r_{1}}=(p-1)\left(\frac{1}{p^{\prime}}-\frac{2}{3}\right)$ and remark that $r_{1}<p^{\prime}$, since $p^{\prime}<\frac{6}{5}$. Applying Theorem 2.1 with $t=r_{1}$, we obtain $u \in L^{q}\left(\mathbb{R}^{2}\right)$ for all $4<q \leq \infty$ such that $\frac{1}{q} \leq \frac{1}{r_{1}}-\frac{2}{3}$. Iterating the procedure we find at each step $u \in L^{q}\left(\mathbb{R}^{2}\right)$ for all $4<q \leq \infty$ such that $\frac{1}{q} \leq \frac{1}{r_{m}}-\frac{2}{3}$, where $r_{m}$ is given by

$$
r_{0}=p^{\prime}, \quad \frac{1}{r_{m}}=(p-1)\left(\frac{1}{r_{m-1}}-\frac{2}{3}\right), m \geq 1 .
$$

It satisfies $\frac{1}{r_{m}}-\frac{1}{r_{m-1}}=2(p-1)^{m}\left(\frac{1}{6}-\frac{1}{p}\right)>0$ and therefore $\frac{1}{r_{m}} \rightarrow \infty$ as $m \rightarrow \infty$. Thus, after finitely many iterations, we obtain $\frac{1}{r_{m}}-\frac{2}{3} \geq \frac{1}{4}$ and the claim follows.

With the help of the above results, we can now give the proof of our first main theorem.

Proof of Theorem 1.1. As remarked above, the regularity was already proved in Lemma 2.4, Restricting to the case where $p>6$, we see that the functions $Q|u|^{p-2}$ and $Q|u|^{p-2} u$ belong to $L^{\frac{6}{5}}\left(\mathbb{R}^{2}\right)$, since $u \in L^{q}\left(\mathbb{R}^{2}\right)$ for all $q>4$ by Lemma 2.4. Thus, Lemma 2.3 with $q=\tilde{q}=\frac{6}{5}, K=\operatorname{Re}(\Phi)$ and $V=Q|u|^{p-2}$ ensures that $u(x)=O\left(|x|^{-\frac{1}{2}}\right)$ as $|x| \rightarrow \infty$. Therefore, $f(x)=O\left(|x|^{-\frac{p-1}{2}}\right)$ as $|x| \rightarrow \infty$. Since $\frac{p-1}{2}>2$, the expansion (5) follows from Proposition 2.2, after taking real parts.

\section{VARIATIONAL SETTING IN THE PLANE AND EXISTENCE OF SOLUTIONS FOR THE NONLINEAR PROBLEM}

In order to prove Theorem 1.3, we extend the dual variational method developed in 11 to the space dimension 2. Let therefore $Q \in L^{\infty}\left(\mathbb{R}^{2}\right) \backslash\{0\}$ be a nonnegative function and consider for $6 \leq p<\infty$ the energy functional

$$
J: L^{p^{\prime}}\left(\mathbb{R}^{2}\right) \rightarrow \mathbb{R}, \quad J(v)=\frac{1}{p^{\prime}} \int_{\mathbb{R}^{2}}|v|^{p^{\prime}} d x-\frac{1}{2} \int_{\mathbb{R}^{2}} Q(x)^{\frac{1}{p}} v(x) \mathbf{R}\left(Q^{\frac{1}{p}} v\right)(x) d x,
$$

In this section, we consider, unless explicitly stated, real-valued functions and denote by $\mathbf{R}$ the real part of the resolvent operator $\mathscr{R}$. Obviously, $J(-v)=J(v)$ for all $v \in L^{p^{\prime}}\left(\mathbb{R}^{2}\right)$. Moreover, one can show that $J$ is of class $C^{1}$ and that every critical point of $J$ corresponds to a solution of (4) in the following way.

A function $v \in L^{p^{\prime}}\left(\mathbb{R}^{2}\right)$ satisfies $J^{\prime}(v)=0$ if and only if it solves the integral equation $|v|^{p^{\prime}-2} v=$ $Q^{\frac{1}{p}} \mathbf{R}\left(Q^{\frac{1}{p}} v\right)$. Setting

$$
u=\mathbf{R}\left(Q^{\frac{1}{p}} v\right) \in L^{p}\left(\mathbb{R}^{2}\right),
$$

it follows that $u=\mathbf{R}\left(Q|u|^{p-2} u\right)$, i.e., $u$ solves (4). Note that, by Lemma 2.4 $u$ is then a strong solution of (2).

As a consequence of Theorem 2.1, the Birman-Schwinger type operator $\mathbf{K}: L^{p^{\prime}}\left(\mathbb{R}^{2}\right) \rightarrow L^{p}\left(\mathbb{R}^{2}\right)$ given by

$$
\mathbf{K} v:=Q^{\frac{1}{p}} \mathbf{R}\left(Q^{\frac{1}{p}} v\right), \quad v \in L^{p^{\prime}}\left(\mathbb{R}^{2}\right),
$$

and appearing in the quadratic part of $J$, is continuous for $6 \leq p<\infty$ and has compactness properties which will be important in the sequel. More precisely, the operator $1_{B} \mathbf{K}: L^{p^{\prime}}\left(\mathbb{R}^{2}\right) \rightarrow L^{p}\left(\mathbb{R}^{2}\right)$ is compact for every bounded and measurable set $B \subset \mathbb{R}^{2}$, and if, in addition, $Q(x) \rightarrow 0$ as $|x| \rightarrow \infty$, then $\mathbf{K}$ itself is compact. Here, $1_{B}$ denotes the characteristic function of the set $B \subset \mathbb{R}^{2}$.

To see this, consider a sequence $\left(v_{n}\right)_{n}$ converging weakly to 0 in $L^{p^{\prime}}\left(\mathbb{R}^{2}\right)$ and choose $R>0$ such that $B \subset B_{R}:=B_{R}(0)$. Due to the continuity of the resolvent, there holds $\mathbf{R}\left(Q^{\frac{1}{p}} v_{n}\right) \rightarrow 0$ in $L^{p}\left(\mathbb{R}^{2}\right)$, and elliptic estimates ensure the boundedness of $\mathbf{R}\left(Q^{\frac{1}{p}} v_{n}\right)$ in the Sobolev space $W^{2, p^{\prime}}\left(B_{R}\right)$. Since $p<\infty$, the embedding $W^{2, p^{\prime}}\left(B_{R}\right) \hookrightarrow L^{p}\left(B_{R}\right)$ is compact, and since $Q$ is bounded and $B \subset B_{R}$, we conclude that $1_{B} \mathbf{K} v_{n}=1_{B} Q^{\frac{1}{p}} \mathbf{R}\left(Q^{\frac{1}{p}} v_{n}\right) \rightarrow 0$, strongly in $L^{p}\left(\mathbb{R}^{2}\right)$, and obtain the first compactness property. In the case where $Q(x) \rightarrow 0$ as $|x| \rightarrow \infty$, the continuity of $\mathbf{R}$ and the estimate

$$
\left\|\left(1-1_{B_{R}}\right) \mathbf{K} v_{n}\right\|_{p} \leq \underset{|x| \geq R}{\operatorname{ess} \sup } Q^{\frac{1}{p}}(x)\left\|\mathbf{R}\left(Q^{\frac{1}{p}} v_{n}\right)\right\|_{p},
$$


which holds for every $R>0$, ensure the convergence $\left\|\left(1-1_{B_{R}}\right) \mathbf{K} v_{n}\right\|_{p} \rightarrow 0$ as $R \rightarrow \infty$, uniformly in $n$. On the other hand, as we have already seen, $\left\|1_{B_{R}} \mathbf{K} v_{n}\right\|_{p} \rightarrow 0$ as $n \rightarrow \infty$, for every $R>0$. Combining these two facts yields the strong convergence $\mathbf{K} v_{n} \rightarrow 0$ in $L^{p}\left(\mathbb{R}^{2}\right)$ and hence the compactness of $\mathbf{K}$.

We start by proving the existence of an unbounded sequence of solutions in the case where $Q(x) \rightarrow 0$ as $|x| \rightarrow \infty$.

Proof of Theorem 1.3(a). The result will follow from the symmetric Mountain Pass Theorem (see 4 for the original work, and 13 for the version we use here) applied to the even functional $J$.

For this, we first show that 0 is a strict local minimum or, more precisely, that $J(v) \geq \delta>0$ for all $\|v\|=\rho$, provided $\rho>0$ is small. Indeed, the operator $\mathbf{K}$ being continuous, there exists a constant $C>0$ such that $\|\mathbf{K} v\|_{p} \leq C\|v\|_{p^{\prime}}$ for all $v \in L^{p^{\prime}}\left(\mathbb{R}^{2}\right)$. Hence, if $\|v\|_{p^{\prime}}=\rho>0$, we obtain

$$
J(v)=\frac{1}{p^{\prime}} \rho^{p^{\prime}}-\frac{1}{2} \int_{\mathbb{R}^{2}} v \mathbf{K} v d x \geq \frac{1}{p^{\prime}} \rho^{p^{\prime}}-\frac{C}{2} \rho^{2}>0
$$

for all $\rho>0$ small enough, since $p^{\prime}<2$.

In the next step, we prove for each integer $m$ the existence of an $m$-dimensional subspace $\mathscr{W}_{m}$ of $L^{p^{\prime}}\left(\mathbb{R}^{2}\right)$ and of a radius $R_{m}>0$ with the property that $J(v) \leq 0$ for all $v \in \mathscr{W}_{m}$ such that $\|v\|_{p^{\prime}} \geq R_{m}$.

Let $m$ be any integer. Since $Q \in L^{\infty}\left(\mathbb{R}^{2}\right) \backslash\{0\}$ is nonnegative, there is a point $x_{0} \in \mathbb{R}^{2}$ of metric density 1 for the set $\{Q>0\}$. Hence, there is $0<\delta<1$ such that

$$
\left|B_{\delta}\left(x_{0}\right) \cap\{Q>0\}\right| \geq \frac{1}{2}\left|B_{\delta}\left(x_{0}\right)\right|
$$

Since $\operatorname{Re}(\Phi)$ is bounded outside of every neighborhood of zero, by (17), and since $\operatorname{Re}(\Phi(x)) \approx \frac{1}{2 \pi} \log \left(\frac{2}{|x|}\right) \rightarrow$ $+\infty$ as $|x| \rightarrow 0$, by (6) , we may also assume that $\delta>0$ satisfies for $\Psi^{*}(\tau):=\inf _{B_{\tau}(0) \backslash\{0\}} \operatorname{Re}(\Phi)$ and $\Psi_{*}(\tau):=\|\operatorname{Re}(\Phi)\|_{L^{\infty}\left(\mathbb{R}^{2} \backslash B_{\tau}(0)\right)}$ the property,

$$
\Psi^{*}\left(\sigma^{m}\right)>(m-1) \Psi_{*}(\sigma) \quad \text { for } \sigma \in(0, \delta] .
$$

Let $\sigma:=\frac{\delta}{4 \sqrt{m}}, \tau:=\frac{1}{2} \sigma^{m}$ and choose $m$ disjoint open balls $B^{1}, \ldots, B^{m} \subset B_{\delta}\left(x_{0}\right)$ as follows. By (23), we can choose $x_{1} \in B_{\delta}\left(x_{0}\right) \cap\{Q>0\}$ and $\tau_{1} \in(0, \tau]$ such that $B^{1}:=B_{\tau_{1}}\left(x_{1}\right) \subset B_{\delta}\left(x_{0}\right)$ and $\left|B^{1} \cap\{Q>0\}\right|>0$. Let now $\omega_{1}:=\left(B_{\delta}\left(x_{0}\right) \cap\{Q>0\}\right) \backslash B_{2 \tau_{1}+\sigma}\left(x_{1}\right)$ and observe that

$$
\left|\omega_{1}\right| \geq \frac{1}{2}\left|B_{\delta}\left(x_{0}\right)\right|-\left|B_{2 \sigma}\left(x_{1}\right)\right| \geq\left(\frac{1}{2}-\frac{1}{4 m}\right) \pi \delta^{2}>0
$$

Thus we may choose $x_{2} \in \omega_{1}$ and $\tau_{2} \in(0, \tau]$ such that $B^{2}:=B_{\tau_{2}}\left(x_{2}\right) \subset B_{\delta}\left(x_{0}\right)$ and $\left|B^{2} \cap\{Q>0\}\right|>0$. Inductively, we let for $2 \leq k \leq m-1, \omega_{k}:=\left(B_{\delta}\left(x_{0}\right) \cap\{Q>0\}\right) \backslash \bigcup_{i=1}^{k} B_{2 \tau_{i}+\sigma}\left(x_{i}\right)$ and remark that

$$
\left|\omega_{k}\right| \geq \frac{1}{2}\left|B_{\delta}\left(x_{0}\right)\right|-\sum_{i=1}^{k}\left|B_{2 \sigma}\left(x_{i}\right)\right| \geq\left(\frac{1}{2}-\frac{k}{4 m}\right) \pi \delta^{2}>0
$$

Therefore, we may choose $x_{k+1} \in \omega_{k}$ and $\tau_{k+1} \in(0, \tau]$ such that $B^{k+1}:=B_{\tau_{k+1}}\left(x_{k+1}\right) \subset B_{\delta}\left(x_{0}\right)$ and $\left|B^{k+1} \cap\{Q>0\}\right|>0$. Notice that, by construction,

$$
\operatorname{diam} B^{i} \leq \sigma^{m} \text { and } \operatorname{dist}\left(B^{i}, B^{j}\right):=\inf \left\{|x-y|: x \in B^{i}, y \in B^{j}\right\} \geq \sigma
$$

for all $i \neq j$. Let us now fix $z_{1}, \ldots, z_{m} \in C_{c}^{\infty}\left(\mathbb{R}^{2}\right)$ such that $z_{i}>0$ in $B^{i}$ and $z_{i}=0$ in $\mathbb{R}^{2} \backslash B^{i}$. We define $\mathscr{W}_{m}$ as the subspace spanned by $z_{1}, \ldots, z_{m}$. Writing any $v \in \mathscr{W}_{m} \backslash\{0\}$ as $v=\sum_{i=1}^{m} a_{i} z_{i}$ with 
$a=\left(a_{1}, \ldots, a_{m}\right) \in \mathbb{R}^{m} \backslash\{0\}$, it follows from (23), (24) and (25) that

$$
\begin{aligned}
\int_{\mathbb{R}^{2}} v \mathbf{K} v d x & =\sum_{i, j=1}^{m} a_{i} a_{j} \int_{B^{i}} \int_{B^{j}} \operatorname{Re}(\Phi)(x-y) Q(x)^{\frac{1}{p}} Q(y)^{\frac{1}{p}} z_{i}(x) z_{j}(y) d x d y \\
& \geq \Psi^{*}\left(\sigma^{m}\right) \sum_{i=1}^{m} a_{i}^{2}\left(\int_{B^{i}} Q(x)^{\frac{1}{p}} z_{i}(x) d x\right)^{2} \\
& -\Psi_{*}(\sigma) \sum_{\substack{i, j=1 \\
i \neq j}}^{m}\left|a_{i}\right|\left|a_{j}\right|\left(\int_{B^{i}} Q(x)^{\frac{1}{p}} z_{i}(x) d x\right)\left(\int_{B^{j}} Q(x)^{\frac{1}{p}} z_{j}(x) d x\right) \\
& \geq \sum_{i=1}^{m}\left(\Psi^{*}\left(\sigma^{m}\right)-(m-1) \Psi_{*}(\sigma)\right) a_{i}^{2}\left(\int_{B^{i}} Q(x)^{\frac{1}{p}} z_{i}(x) d x\right)^{2}>0
\end{aligned}
$$

(cf. [11, Lemma 5.1]). From the continuity of $\mathbf{K}$, we obtain

$$
\mu_{m}:=\inf _{v \in \mathscr{W}_{m},\|v\|_{p^{\prime}}=1} \int_{\mathbb{R}^{2}} v \mathbf{K} v d x>0,
$$

and therefore,

$$
J(v)=\frac{\|v\|_{p^{\prime}}^{p^{\prime}}}{p^{\prime}}-\frac{1}{2} \int_{\mathbb{R}^{2}} v \mathbf{K} v d x \leq\|v\|_{p^{\prime}}^{p^{\prime}}\left(\frac{1}{p^{\prime}}-\frac{1}{2}\|v\|_{p^{\prime}}^{2-p^{\prime}} \mu_{m}\right) \quad \text { for } v \in \mathscr{W}_{m} .
$$

Since $p^{\prime}<2$, the assertion follows by choosing $R_{m}>0$ large enough.

The last step to apply the Mountain Pass Theorem is to check the Palais-Smale condition for $J$. Let $\left(v_{n}\right)_{n} \subset L^{p^{\prime}}\left(\mathbb{R}^{2}\right)$ be a Palais-Smale sequence for $J$, i.e., $\left(J\left(v_{n}\right)\right)_{n}$ is bounded and $J^{\prime}\left(v_{n}\right) \rightarrow 0$ in $L^{p^{\prime}}\left(\mathbb{R}^{2}\right)^{*}$ as $n \rightarrow \infty$, we need to show that $\left(v_{n}\right)_{n}$ has a subsequence converging strongly in $L^{p^{\prime}}\left(\mathbb{R}^{2}\right)$. Here, the assumption $Q(x) \rightarrow 0,|x| \rightarrow \infty$, will come into play.

First observe that since

$$
J\left(v_{n}\right)=\left(\frac{1}{p^{\prime}}-\frac{1}{2}\right)\left\|v_{n}\right\|_{p^{\prime}}^{p^{\prime}}+\frac{1}{2} J^{\prime}\left(v_{n}\right) v_{n} \geq\left(\frac{1}{p^{\prime}}-\frac{1}{2}\right)\left\|v_{n}\right\|_{p^{\prime}}^{p^{\prime}}-\frac{1}{2}\left\|J^{\prime}\left(v_{n}\right)\right\|\left\|_{*}\right\| v_{n} \|_{p^{\prime}}
$$

and $1<p^{\prime}<2$, the sequence $\left(v_{n}\right)_{n}$ is bounded in $L^{p^{\prime}}\left(\mathbb{R}^{2}\right)$. Hence, there is $v \in L^{p^{\prime}}\left(\mathbb{R}^{2}\right)$ such that, up to a subsequence, $v_{n} \rightarrow v$ in $L^{p^{\prime}}\left(\mathbb{R}^{2}\right)$ and $\|v\|_{p^{\prime}} \leq \liminf _{n \rightarrow \infty}\left\|v_{n}\right\|_{p^{\prime}}$. From the convexity of the function $t \mapsto|t|^{p^{\prime}}$ and the compactness of the operator $\mathbf{K}$, guaranteed by the assumption that $Q(x) \rightarrow 0$ as $|x| \rightarrow \infty$, it follows that $\lim _{n \rightarrow \infty}\left\|v_{n}\right\|_{p^{\prime}}=\|v\|_{p^{\prime}}$, and therefore $v_{n} \rightarrow v$ strongly in $L^{p^{\prime}}\left(\mathbb{R}^{2}\right)$. Hence $J$ satisfies the Palais-Smale condition.

Since the hypotheses of the symmetric Mountain Pass Theorem are fulfilled, there exists a sequence of pairs $\left\{ \pm v_{n}\right\}$ of nontrivial critical points of $J$ with $J\left(v_{n}\right) \rightarrow \infty$ and thus, $\left\|v_{n}\right\|_{p^{\prime}} \rightarrow \infty$ as $n \rightarrow \infty$. Setting $u_{n}:=\mathbf{R}\left(Q^{\frac{1}{p}} v_{n}\right)$ and using Lemma 2.4 concludes the proof.

In the case where $Q$ is periodic, the Palais-Smale condition is in general not satisfied. A crucial ingredient in the proof of Theorem 1.3(b) will be the following nonvanishing property of the resolvent, analogue to [11, Theorem 3.1] and reminiscent of the Lions compactness lemma [21, II. Lemma I.1]. It will allow us to obtain the existence, up to translations, of a nontrivial weak limit for a Palais-Smale sequence of $J$. We prove the result in a more general form than needed, since we believe that it will be useful for the study of complex-valued solutions of (2) also.

Theorem 3.1. Let $6<p<\infty$ and consider a bounded sequence $\left(v_{n}\right)_{n} \subset L^{p^{\prime}}\left(\mathbb{R}^{2}\right)$ satisfying

$$
\limsup _{n \rightarrow \infty}\left|\int_{\mathbb{R}^{2}} v_{n} \mathscr{R} v_{n} d x\right|>0 .
$$

Then there exists $R>0, \zeta>0$ and $\left(x_{n}\right)_{n} \subset \mathbb{R}^{2}$ such that, up to a subsequence,

$$
\int_{B_{R}\left(x_{n}\right)}\left|v_{n}\right|^{p^{\prime}} d x \geq \zeta \quad \text { for all } n \text {. }
$$


Proof. Recall the decomposition of the fundamental solution $\Phi=\Phi_{1}+\Phi_{2}$ introduced in (9), and the estimates (10) and (11):

$$
\left|\Phi_{1}(x)\right| \leq C_{0}(1+|x|)^{-\frac{1}{2}} \quad \text { and } \quad\left|\Phi_{2}(x)\right| \leq C_{0} \min \left\{1+|\log | x||,|x|^{-3}\right\} .
$$

The proof of the theorem consists in three claims. We first prove a variant of the conclusion with $\Phi_{2}$ in place of $\Phi$ and for Schwartz functions. Next, a decay estimate for the convolution with $\Phi_{1}$ outside larger and larger balls is established. It is used in the third step to obtain the conclusion of the theorem for $\Phi_{1}$ in place of $\Phi$, again for Schwartz functions.

Claim 1: Let $2<p<\infty$, and $\left(v_{n}\right)_{n} \subset \mathscr{S}\left(\mathbb{R}^{2}\right)$ bounded in $L^{p^{\prime}}\left(\mathbb{R}^{2}\right)$ satisfy

$$
\sup _{y \in \mathbb{R}^{2}} \int_{B_{\rho}(y)}\left|v_{n}\right|^{p^{\prime}} d x \rightarrow 0, \quad \text { as } n \rightarrow \infty, \quad \text { for all } \rho>0 \text {. }
$$

Then $\int_{\mathbb{R}^{2}} v_{n}\left[\Phi_{2} * v_{n}\right] d x \rightarrow 0$ as $n \rightarrow \infty$.

For the proof, let $A_{R}:=\left\{x \in \mathbb{R}^{2}: \frac{1}{R} \leq|x| \leq R\right\}$ and $D_{R}:=\mathbb{R}^{2} \backslash A_{R}$ for $R>1$, and first remark that (11) gives $\Phi_{2} \in L^{t}\left(\mathbb{R}^{2}\right)$ for all $1 \leq t<\infty$. Hence, Young's inequality implies

$$
\sup _{n \in \mathbb{N}}\left|\int_{\mathbb{R}^{2}} v_{n}\left[\left(1_{D_{R}} \Phi_{2}\right) * v_{n}\right] d x\right| \leq\left\|\Phi_{2}\right\|_{L^{\frac{p}{2}}\left(D_{R}\right)} \sup _{n \in \mathbb{N}}\left\|v_{n}\right\|_{L^{p^{\prime}\left(\mathbb{R}^{2}\right)}}^{2} \rightarrow 0, \text { as } R \rightarrow \infty,
$$

since $2<p<\infty$. Next, decomposing $\mathbb{R}^{2}$ into disjoint squares $\left\{Q_{\ell}\right\}_{\ell \in \mathbb{N}}$ of side length $R$, and considering for each $\ell$ the square $Q_{\ell}^{\prime}$ with the same center as $Q_{\ell}$ but with side length $3 R$, we obtain by an estimate similar to [1, pp. 109-110],

$$
\begin{aligned}
& \left|\int_{\mathbb{R}^{2}} v_{n}\left[\left(1_{A_{R}} \Phi_{2}\right) * v_{n}\right] d x\right| \leq \sum_{\ell=1}^{\infty} \int_{Q_{\ell}}\left(\int_{\frac{1}{R}<|x-y|<R}\left|\Phi_{2}(x-y) \| v_{n}(x)\right|\left|v_{n}(y)\right| d y\right) d x \\
& \leq C R^{\frac{4}{p}}(1+\log R) \sum_{\ell=1}^{\infty}\left(\int_{Q_{\ell}^{\prime}}\left|v_{n}(x)\right|^{p^{\prime}} d x\right)^{\frac{2}{p^{\prime}}} \\
& \quad \leq C R^{\frac{4}{p}}(1+\log R)\left[\sup _{\ell \in \mathbb{N}} \int_{Q_{\ell}^{\prime}}\left|v_{n}(x)\right|^{p^{\prime}} d x\right]^{\frac{2}{p^{\prime}}-1} \sum_{\ell=1}^{\infty} \int_{Q_{\ell}^{\prime}}\left|v_{n}(x)\right|^{p^{\prime}} d x \\
& \leq C R^{\frac{4}{p}}(1+\log R)\left[\sup _{y \in \mathbb{R}^{2}} \int_{B_{3 R \sqrt{2}}(y)}\left|v_{n}(x)\right|^{p^{\prime}} d x\right]^{\frac{2}{p^{\prime}}-1}\left\|v_{n}\right\|_{p^{\prime}}^{p^{\prime}}
\end{aligned}
$$

The assumption (28) therefore gives $\int_{\mathbb{R}^{2}} v_{n}\left[\left(1_{A_{R}} \Phi_{2}\right) * v_{n}\right] d x \rightarrow 0$, as $n \rightarrow \infty$, for every $R>0$. Combining this with (29), the claim follows.

Claim 2: Let $6<p \leq \infty, \lambda_{p}:=\frac{1}{2}-\frac{3}{p}>0$ and $M_{R}:=\mathbb{R}^{N} \backslash B_{R}$ for $R>0$. Then there exists a constant $C>0$ such that, for all $R \geq 1$ and $f \in \mathscr{S}\left(\mathbb{R}^{2}\right)$ with $\operatorname{supp} \widehat{f} \subset\left\{\xi:|| \xi|-1| \leq \frac{1}{2}\right\}$,

$$
\left\|\left[1_{M_{R}} \Phi_{1}\right] * f\right\|_{p} \leq C R^{-\lambda_{p}}\|f\|_{p^{\prime}} .
$$

Since $\Phi_{1}$ is bounded, it suffices to prove the assertion for $R \geq 4$. For this, let us replace in the decomposition (13) the function $\Phi_{1}$ by $P_{R}:=1_{M_{R}} \Phi_{1}$. Then,

$$
P_{R} * \varphi=\sum_{j=\left[\log _{2} R\right]}^{\infty} Q^{j} \quad \text { with } Q^{j}:=\left(P_{R} \varphi_{j}\right) * \varphi \text { for } j \in \mathbb{N},
$$

using the fact that $P_{R} \varphi_{j}=0$ for all $j$ such that $2^{j+1} \leq R$. Since the asymptotic behavior of $\Phi_{1}$ and $P_{R}$ are identical, the arguments used there give as in (15),

$$
\left\|Q^{j} * f\right\|_{2} \leq C 2^{\frac{j}{2}}\|f\|_{\frac{6}{5}} \quad \text { for all } f \in \mathscr{S}\left(\mathbb{R}^{2}\right), j \geq\left[\log _{2} R\right]
$$

with a constant $C>0$ independent of $j$ and $R$. By duality and interpolation

$$
\left\|Q^{j} * f\right\|_{3} \leq C 2^{\frac{j}{2}}\|f\|_{\frac{4}{3}} \quad \text { for all } f \in \mathscr{S}\left(\mathbb{R}^{2}\right), j \geq\left[\log _{2} R\right] .
$$

Interpolating this last estimate with the $L^{1}-L^{\infty}$ estimate

$$
\left\|Q^{j} * f\right\|_{\infty} \leq C 2^{-\frac{j}{2}}\|f\|_{1}, \quad f \in \mathscr{S}\left(\mathbb{R}^{2}\right), j \geq\left[\log _{2} R\right],
$$


similar to (14), we obtain for $p \geq 3$,

$$
\left\|Q^{j} * f\right\|_{p} \leq C 2^{j\left(\frac{3}{p}-\frac{1}{2}\right)}\|f\|_{p^{\prime}}=C 2^{-j \lambda_{p}}\|f\|_{p^{\prime}} \quad \text { for all } f \in \mathscr{S}\left(\mathbb{R}^{2}\right), j \geq\left[\log _{2} R\right] .
$$

Since $P_{R} * f=\frac{1}{2 \pi}\left(P_{R} * \varphi\right) * f$ for all $f \in \mathscr{S}\left(\mathbb{R}^{2}\right)$ with supp $\widehat{f} \subset\left\{\xi:|| \xi|-1| \leq \frac{1}{2}\right\}$, and since $\lambda_{p}>0$ for $p>6$, we conclude that for all such $f$ and all $p>6$,

$$
\left\|\left[1_{M_{R}} \Phi_{1}\right] * f\right\|_{p}=\frac{1}{2 \pi}\left\|\left(P_{R} * \varphi\right) * f\right\|_{p} \leq C\|f\|_{p^{\prime}} \sum_{j=\left[\log _{2} R\right]}^{\infty} 2^{-j \lambda_{p}} \leq C R^{-\lambda_{p}}\|f\|_{p^{\prime}} .
$$

The claim is proved.

Claim 3: Let $6<p \leq \infty$ and suppose that $\left(v_{n}\right)_{n} \subset \mathscr{S}\left(\mathbb{R}^{2}\right)$ is a bounded sequence in $L^{p^{\prime}}\left(\mathbb{R}^{2}\right)$ such that (28) holds. Then $\int_{\mathbb{R}^{2}} v_{n}\left[\Phi_{1} * v_{n}\right] d x \rightarrow 0$ as $n \rightarrow \infty$.

To prove the claim, fix a radial function $\chi \in \mathscr{S}\left(\mathbb{R}^{2}\right)$ such that $\hat{\chi} \in C_{c}^{\infty}\left(\mathbb{R}^{2}\right)$ is radial, $0 \leq \hat{\chi} \leq 1$, $\widehat{\chi}(\xi)=1$ for ||$\xi|-1| \leq \frac{1}{4}$ and $\widehat{\chi}(\xi)=0$ for ||$\xi|-1| \geq \frac{1}{2}$. Moreover, let $w_{n}:=\chi * v_{n}$. We then have $\Phi_{1} * v_{n}=\frac{1}{2 \pi} \Phi_{1} * w_{n}$, since supp $\widehat{w_{n}} \subset\left\{\xi:|| \xi|-1| \leq \frac{1}{2}\right\}$. Hence, the decomposition

$$
\int_{\mathbb{R}^{2}} v_{n}\left[\Phi_{1} * v_{n}\right] d x=\frac{1}{2 \pi} \int_{\mathbb{R}^{2}} v_{n}\left[\left(1_{B_{R}} \Phi_{1}\right) * w_{n}\right] d x+\frac{1}{2 \pi} \int_{\mathbb{R}^{2}} v_{n}\left[\left(1_{M_{R}} \Phi_{1}\right) * w_{n}\right] d x
$$

holds for all $n$ and using Claim 2 we obtain

$$
\sup _{n \in \mathbb{N}}\left|\int_{\mathbb{R}^{2}} v_{n}\left[\left(1_{M_{R}} \Phi_{1}\right) * w_{n}\right] d x\right| \rightarrow 0, \quad \text { as } R \rightarrow \infty .
$$

Moreover, using the disjoint squares $Q_{\ell}$ and $Q_{\ell}^{\prime}$ of the proof of Claim 1 (cf. also [11, Lemma 4.3]) and the assumption (28), we obtain

$$
\lim _{n \rightarrow \infty} \int_{\mathbb{R}^{2}} v_{n}\left[\left(1_{B_{R}} \Phi_{1}\right) * w_{n}\right] d x=0 \quad \text { for every } R>0 .
$$

Combining this with (30), the claim follows.

Arguing by contradiction, we obtain from Claim 1 and Claim 3 the conclusion of the theorem for sequences $\left(v_{n}\right)_{n} \subset \mathscr{S}\left(\mathbb{R}^{2}\right)$, bounded in the $L^{p^{\prime}}$-norm. Since the bilinear form $\int_{\mathbb{R}^{2}} v \mathscr{R} v d x$ is continuous on $L^{p^{\prime}}\left(\mathbb{R}^{2}\right)$ and since the property (27) is stable under approximation in the $L^{p^{\prime}}\left(\mathbb{R}^{2}\right)$-norm, the conclusion follows by density.

We end this paper by giving the proof of the part (b) of our second main result, showing the existence of a nontrivial solution pair for (4).

Proof of Theorem 1.3(b). Consider the set of paths $\Gamma=\left\{\gamma \in C\left([0,1], L^{p^{\prime}}\left(\mathbb{R}^{2}\right)\right): \gamma(0)=0, J(\gamma(1))<0\right\}$ and the energy level

$$
c=\inf _{\gamma \in \Gamma} \max _{t \in[0,1]} J(\gamma(t)) .
$$

Notice that $\Gamma \neq \varnothing$ and $c>0$, since 0 is a strict local minimum of $J$ and there are $v \in L^{p^{\prime}}\left(\mathbb{R}^{2}\right)$ such that $J(v)<0$. Indeed, the proof of Theorem [1.3(a) gives these facts without any decay assumption on $Q$. Using the standard deformation lemma (see [26. Lemma 2.3]), we obtain the existence of a Palais-Smale sequence $\left(v_{n}\right)_{n} \subset L^{p^{\prime}}\left(\mathbb{R}^{2}\right)$ such that $J\left(v_{n}\right) \rightarrow c$, as $n \rightarrow \infty$. By (26), this sequence is bounded and therefore, as $n \rightarrow \infty$,

$$
\int_{\mathbb{R}^{2}} Q^{\frac{1}{p}} v_{n} \mathbf{R}\left(Q^{\frac{1}{p}} v_{n}\right) d x=\left(\frac{1}{p^{\prime}}-\frac{1}{2}\right)^{-1}\left(J\left(v_{n}\right)-\frac{1}{p^{\prime}} J^{\prime}\left(v_{n}\right) v_{n}\right) \rightarrow \frac{2 p^{\prime} c}{2-p^{\prime}}>0 .
$$

Consequently, Theorem 3.1 gives $R, \zeta>0$ and $\left(x_{n}\right)_{n} \subset \mathbb{R}^{2}$ satisfying (27), up to a subsequence. Making $R$ larger we may assume $x_{n} \in \mathbb{Z}^{2}$ for all $n$. Since $Q$ is $\mathbb{Z}^{2}$-periodic, the functional $J$ is invariant under $\mathbb{Z}^{2}$-translations and setting $w_{n}:=v_{n}\left(\cdot-x_{n}\right)$ we find that $\left(w_{n}\right)_{n}$ is also a bounded Palais-Smale sequence for $J$. Going to a subsequence, $w_{n} \rightarrow w \in L^{p^{\prime}}\left(\mathbb{R}^{2}\right)$. Moreover, if $\varphi \in C_{c}^{\infty}\left(\mathbb{R}^{2}\right)$,

$$
\begin{aligned}
& \left|\int_{\mathbb{R}^{2}}\left(\left|w_{n}\right|^{p^{\prime}-2} w_{n}-\left|w_{m}\right|^{p^{\prime}-2} w_{m}\right) \varphi d x\right| \\
& \leq\left\|J^{\prime}\left(w_{n}\right)-J^{\prime}\left(w_{m}\right)\right\|_{*}\|\varphi\|_{p^{\prime}}+\left\|1_{B} \mathbf{K}\left(w_{n}-w_{m}\right)\right\|\left\|_{p}\right\| \varphi \|_{p^{\prime}} \rightarrow 0,
\end{aligned}
$$


as $m, n \rightarrow \infty$, thanks to the compactness of $1_{B} \mathbf{K}$, where $B \subset \mathbb{R}^{2}$ contains $\operatorname{supp}(\varphi)$. Since $C_{c}^{\infty}\left(\mathbb{R}^{2}\right)$ is dense in $L^{p^{\prime}}\left(\mathbb{R}^{2}\right)$, we infer that for all bounded and measurable $B \subset \mathbb{R}^{2},\left(1_{B}\left|w_{n}\right|^{p^{\prime}-2} w_{n}\right)_{n}$ is a Cauchy sequence in $L^{p}\left(\mathbb{R}^{2}\right)$ and thus,

$$
1_{B}\left|w_{n}\right|^{p^{\prime}-2} w_{n} \rightarrow 1_{B}|w|^{p^{\prime}-2} w \text { as } n \rightarrow \infty, \quad \text { strongly in } L^{p}\left(\mathbb{R}^{2}\right) .
$$

Recalling (27), we see that

$$
\int_{B_{R}(0)}|w|^{p^{\prime}} d x=\lim _{n \rightarrow \infty} \int_{B_{R}(0)}\left|w_{n}\right|^{p^{\prime}} d x \geq \zeta>0
$$

and consequently, $w \neq 0$. In addition, for all $\varphi \in C_{c}^{\infty}\left(\mathbb{R}^{2}\right)$, we obtain

$$
\begin{aligned}
J^{\prime}(w) \varphi & =\int_{\mathbb{R}^{2}}|w|^{p^{\prime}-2} w \varphi-\int_{\mathbb{R}^{2}} \varphi \mathbf{K} w d x \\
& =\lim _{n \rightarrow \infty} \int_{\mathbb{R}^{2}}\left|w_{n}\right|^{p^{\prime}-2} w_{n} \varphi-\int_{\mathbb{R}^{2}} \varphi \mathbf{K} w_{n} d x=\lim _{n \rightarrow \infty} J^{\prime}\left(w_{n}\right) \varphi=0,
\end{aligned}
$$

and we conclude that $J^{\prime}(w)=0$. Hence, $w$ is a nontrivial critical point of $J$ and, applying Lemma 2.4. the theorem follows.

\section{ACKNOWLEDGEMENTS}

This research is supported by the Grant WE 2821/5-1 of the Deutsche Forschungsgemeinschaft (DFG). The author would like to thank Tobias Weth for suggesting the study of the 2-dimensional case and for his valuable remarks on a preliminary version of the manuscript.

\section{REFERENCES}

[1] S. Alama and Y. Y. Li. Existence of solutions for semilinear elliptic equations with indefinite linear part. J. Differential Equations, 96(1):89-115, 1992.

[2] P. Alsholm and G. Schmidt. Spectral and scattering theory for Schrödinger operators. Arch. Rational Mech. Anal., 40:281-311, 1970/1971.

[3] A. Ambrosetti and A. Malchiodi. Nonlinear analysis and semilinear elliptic problems, volume 104 of Cambridge Studies in Advanced Mathematics. Cambridge University Press, Cambridge, 2007.

[4] A. Ambrosetti and P. H. Rabinowitz. Dual variational methods in critical point theory and applications. J. Functional Analysis, 14:349-381, 1973.

[5] H. Berestycki, T. Gallouët, and O. Kavian. Équations de champs scalaires euclidiens non linéaires dans le plan. C. R. Acad. Sci. Paris Sér. I Math., 297(5):307-310, 1983.

[6] H. Berestycki and P.-L. Lions. Nonlinear scalar field equations. I. Existence of a ground state. Arch. Rational Mech. Anal., 82(4):313-345, 1983.

[7] H. Berestycki and P.-L. Lions. Nonlinear scalar field equations. II. Existence of infinitely many solutions. Arch. Rational Mech. Anal., 82(4):347-375, 1983.

[8] D. Colton and R. Kress. Inverse acoustic and electromagnetic scattering theory, volume 93 of Applied Mathematical Sciences. Springer-Verlag, Berlin, 1992.

[9] G. Evéquoz. A dual approach in Orlicz spaces for the nonlinear Helmholtz equation. Z. Angew. Math. Phys., 66:2995$3015,2015$.

[10] G. Evéquoz and T. Weth. Real solutions to the nonlinear Helmholtz equation with local nonlinearity. Arch. Rat. Mech. Anal., 211(2):359-388, 2014.

[11] G. Evéquoz and T. Weth. Dual variational methods and nonvanishing for the nonlinear Helmholtz equation. Adv. in Math., 280:690-728, 2015.

[12] I. M. Gel'fand and G. E. Shilov. Generalized functions. Vol. 1. Academic Press [Harcourt Brace Jovanovich Publishers], New York, 1964 [1977]. Properties and operations, Translated from the Russian by Eugene Saletan.

[13] N. Ghoussoub. Duality and perturbation methods in critical point theory, volume 107 of Cambridge Tracts in Mathematics. Cambridge University Press, Cambridge, 1993. With appendices by David Robinson.

[14] D. Gilbarg and N. S. Trudinger. Elliptic partial differential equations of second order. Classics in Mathematics. SpringerVerlag, Berlin, 2001. Reprint of the 1998 edition.

[15] S. Gutiérrez. Non trivial $L^{q}$ solutions to the Ginzburg-Landau equation. Math. Ann., 328(1-2):1-25, 2004.

[16] E. Jalade. Inverse problem for a nonlinear Helmholtz equation. Ann. Inst. H. Poincaré Anal. Non Linéaire, 21(4):517531,2004

[17] T. Kato. Growth properties of solutions of the reduced wave equation with a variable coefficient. Comm. Pure Appl. Math., 12:403-425, 1959.

[18] C. E. Kenig, A. Ruiz, and C. D. Sogge. Uniform Sobolev inequalities and unique continuation for second order constant coefficient differential operators. Duke Math. J., 55(2):329-347, 1987.

[19] I. Kuzin and S. Pohozaev. Entire solutions of semilinear elliptic equations. Progress in Nonlinear Differential Equations and their Applications, 33. Birkhäuser Verlag, Basel, 1997. 
[20] N. N. Lebedev. Special functions and their applications. Dover Publications Inc., New York, 1972. Revised edition, translated from the Russian and edited by Richard A. Silverman, Unabridged and corrected republication.

21] P.-L. Lions. The concentration-compactness principle in the calculus of variations. The locally compact case. I, II. Ann. Inst. H. Poincaré Anal. Non Linéaire, 1:109-145, 223-283, 1984.

[22] P. H. Rabinowitz. Minimax methods in critical point theory with applications to differential equations, volume 65 of CBMS Regional Conference Series in Mathematics. Published for the Conference Board of the Mathematical Sciences, Washington, DC; by the American Mathematical Society, Providence, RI, 1986.

[23] E. M. Stein and G. Weiss. Introduction to Fourier analysis on Euclidean spaces. Princeton University Press, Princeton, N.J., 1971. Princeton Mathematical Series, No. 32

[24] M. Struwe. Variational methods. Applications to nonlinear partial differential equations and Hamiltonian systems, volume 34 of Ergebnisse der Mathematik und ihrer Grenzgebiete. 3. Folge. A Series of Modern Surveys in Mathematics. Springer-Verlag, Berlin, fourth edition, 2008.

[25] P. A. Tomas. A restriction theorem for the Fourier transform. Bull. Amer. Math. Soc., 81:477-478, 1975.

[26] M. Willem. Minimax theorems. Progress in Nonlinear Differential Equations and their Applications, 24. Birkhäuser Boston Inc., Boston, MA, 1996

[27] C. Zemach and F. Odeh. Uniqueness of radiative solutions to the Schroedinger wave equation. Arch. Rational Mech. Anal., 5:226-237, 1960.

Institut für Mathematik, Johann Wolfgang Goethe-Universität, Robert-Mayer-Str. 10, 60054 Frankfurt am Main, Germany

E-mail address: evequoz@math.uni-frankfurt.de 